
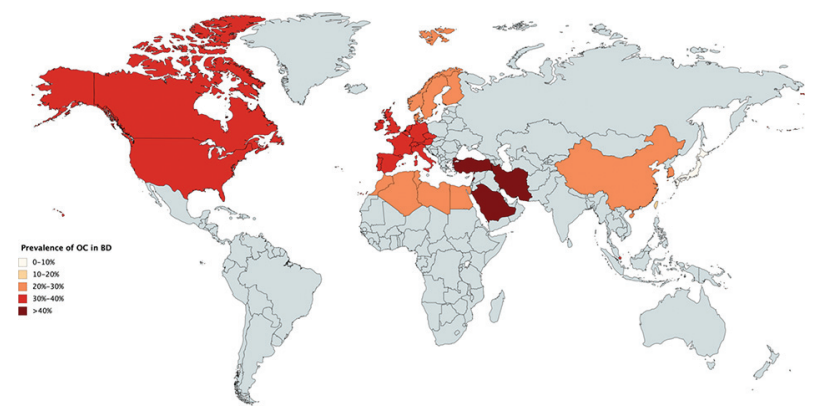

Disclosure of Interests: Matthew Turk: None declared, Jacqueline Hayworth: None declared, Tatiana Nevskaya: None declared, Janet Pope Consultant for: Eli Lilly and Company

DOI: 10.1136/annrheumdis-2019-eular.500

\section{THU0693 INPATIENT PREVALENCE, EXPENDITURES AND COMORBIDITIES OF TAKAYASU'S ARTERITIS: A PROPENSITY-MATCHED COHORT STUDY}

Patompong Ungprasert ${ }^{1}$, Karn Wijarnpreecha. ${ }^{2}$, Wisit Cheungpasitporn ${ }^{3}$, Charat Thongprayoon ${ }^{4}$. ${ }^{1}$ Faculty of Medicine Siriraj Hospital, Mahidol University, Clinical Epidemiology Unit, Department of Research and Development, Bangkok, Thailand; ${ }^{2}$ Mayo Clinic Florida, Medicine, Jacksonville, United States of America; ${ }^{3}$ University of Mississippi Medical Center, Medicine, Jackson, United States of America; ${ }^{4}$ Mayo Clinic Rochester, Medicine, Rochester, United States of America

Background: Takayasu's arteritis (TAK) was first described in Japan. Since then, the disease has been extensively studied in Japan and other Asian countries [1]. However, little is known about the characteristics, inpatient burden, expenditures and comorbidities of TAK in the United States (US).

Objectives: To investigate the inpatient prevalence, expenditures and comorbidities of patients with TAK in the US.

Methods: Patients with TAK were identified within the Nationwide Inpatient Sample (NIS) database of the years 2013-2014 using ICD-9 diagnostic code. NIS is a publicly available inpatient database that contained data of over 7 million hospital stays, which are a $20 \%$ stratified sample of over 4,000 non-federal acute care hospitals from more than 40 states of the US. Data on patient characteristics, comorbidities, resource utilization and expenditures was collected. A propensity-matched cohort of patients without TAK was also created from the same database to serve as comparators for the analysis of comorbidities. Inpateint prevalence of TAK was calculated using all admissions in the NIS database as denominator. Odds ratios (OR) comapring the prevalence of comorbities between cases with TAK and propensity-matched controls without TAK were calculated.

Results: A total of 2,840 patients with TAK were identified from the database, corresponding to an inpatient prevalence of 4.6 cases per 100,000 admissions. The 5 main reasons for admission in patients with TAK were as follows; chest pain (17\%), acute myocardial infarction (16\%), stroke $(14 \%)$, sepsis $(14 \%)$ and pneumonia $(11 \%)$. Compared to the propensitymatched cohort of patients without TAK, patients with TAK were found to have significantly increased odds of stroke, aortic aneurysm, aortic valvulopathy and peripheral vascular disease. TAK was also associated with increased use of some procedures (Table 1). However, the mortality was not significantly different (adjusted OR: $1.44,95 \%$ Cl: 0.58 - 3.61, $\mathrm{p}=0.43$ ). After adjusting for confounders, patients with TAK displayed a mean additional $\$ 11,275(95 \% \mathrm{Cl}, \$ 4,946-\$ 17,603)$ for total hospital costs (the amount of money invested by each institution in providing patient care) and a mean additional $\$ 45,305 \quad(95 \% \mathrm{Cl}, \quad \$ 23,063-$ $\$ 67,546$ ) for total hospitalization charges (the amount of money that each hospital billed for providing its service on each case) when compared to patients without TAK.

Conclusion: The inpatient prevalence of TAK was higher than what would be expected from the overall incidence. The mean total hospital costs and total hospitalization charges for patients with TAK were higher than patients without TAK. Analysis of comorbidities found significantly higher odds of several vascular comorbidities compared to a propensitymatched cohort of patients without TAK.

\section{REFERENCES:}

[1] Onen F, Akkoc N. Epidemiology of Takayasu arteritis. Presse Med 2017;46:e197-e203
Table 1. Adjusted ORs comparing the prevalence of comorbidities and use of procedures between patients with TAK versus patients without TAK

\begin{tabular}{lccc}
\hline & Adjusted odds ratio & $95 \% \mathrm{Cl}$ & $\boldsymbol{p}$-value \\
\hline Comorbidities & & & \\
Stroke & 4.66 & $2.10-10.31$ & $<0.01$ \\
Aortic aneurysm & 40.76 & $9.13-181.7$ & $<0.01$ \\
Peripheral vascular disease & 2.01 & $1.22-3.32$ & $<0.01$ \\
Acute myocardial infarction & 2.13 & $0.56-8.13$ & 0.27 \\
Aortic valvulopathy & 4.92 & $2.09-11.55$ & $<0.01$ \\
Chronic kidney disease & 1.22 & $0.63-2.63$ & 0.57 \\
Procedures & & & \\
Aortic valve replacement & 1.39 & $0.42-4.60$ & 0.59 \\
Peripheral vascular intervention & 4.41 & $1.61-12.10$ & $<0.01$ \\
Arteriography & 6.82 & $2.79-16.68$ & $<0.01$ \\
MRI use & 2.60 & $0.42-16.28$ & 0.31 \\
\hline
\end{tabular}

Disclosure of Interests: None declared

DOI: 10.1136/annrheumdis-2019-eular.493

\section{THU0694 BRAZILIAN SJÖGREN'S SYNDROME REGISTRY (BRASS): A LARGE BRAZILIAN MULTICENTRIC COHORT OF PRIMARY SJÖGREN'S SYNDROME}

Valeria Valim ${ }^{1}$, Samira Tatiyama Miyamoto ${ }^{1}$, Maria Lúcia Lemos Lopes ${ }^{2}$, Aysa Cesar Pinheiro ${ }^{3}$, Aysa Cesar Pinheiro ${ }^{4}$, Leandro Augusto Tanure ${ }^{5}$, Fabiola Reis Oliveira ${ }^{6}, V^{2}$ anessa $\mathrm{Hax}^{7}$, Aiessa Zanchett Fedrigo ${ }^{8}$, Henrique

Pereira Sampaio ${ }^{9}$, Roberta de Almeida Pernambuco ${ }^{10}$, Érica Vieira Serrano ${ }^{1}$, Sabrina Zanardi Machado ${ }^{1}$, Virginia Fernandes Moça Trevisani ${ }^{11}$, Brazilian Committee on Sjögren's syndrome of Brazilian Society of Rheumatology.

${ }^{1}$ Universidade Federal do Espírito Santo, Vitória, Brazil; ${ }^{2}$ Universidade Federal de Porto Alegre, Porto Alegre, Brazil; ${ }^{3}$ Universidade Federal de Pernambuco, Pernambuco, Brazil; ${ }^{4}$ Universidade Federal de Uberlândia, Uberlândia, Brazil; ${ }^{5}$ Hospital das Clínicas da Universidade Federal de Minas Gerais, Belo Horizonte, Brazil; ${ }^{6}$ Hospital das Clínicas de Ribeirão Preto, Ribeirão Preto, Brazil; ${ }^{7}$ Hospital de Clínicas de Porto Alegre, Porto Alegre, Brazil; ${ }^{8}$ Hospital Evangélico de Curitiba, Curitiba, Brazil; ${ }^{9}$ Faculdade de Medicina de Botucatu - UNESP, Botucatu, Brazil;

${ }^{10}$ Instituto de Assistência Médica ao Servidor Público Estadual, São Paulo, Brazil;

${ }^{11}$ Universidade Federal de São Paulo and Universidade de Santo Amaro., São Paulo, Brazil

Background: Primary Sjögren's syndrome (pSS) is an orphan systemic autoimmune disease with no treatment based on evidence ${ }^{1}$. There is an international effort for multicentric registries for getting information about phenotypes, complication, response of treatment, and biobank consortium. Objectives: To describe the creation of the Brazilian Sjögren's Syndrome Registry (BRASS) and present the preliminary data.

Methods: BRASS is supported by Brazilian Society of Rheumatology (SBR) and is including patients from all regions of the country. Recruitment started in 2018 and is due to be completed in 2024. We are including patients with pSS according to AECG 2002 or ACR-EULAR 2016 classification criteria. All patients are being assessed for disease activity (ESSDAl), disease damage (SSDDI), symptoms assessment (ESSPRI), fatigue (FACIT-Fatigue), anxiety and depression (HADS), sleepiness (Epworth Sleepiness Scale), physical activity (IPAQ-SF) and quality of life (EQ-5D). In addition, demographics, immunological tests, unstimulated whole salivary flow (UWSF), salivary gland biopsy (SGB), comorbidities, treatment and complications such as cancer and cardiovascular risk assessment are being collected.

Results: There are currently 10 centers across the Brazil and 248 patients were evaluated until now. Most patients were female, white $(45 \%)$ or mixed $(38.3 \%)$, with the mean of the disease duration of 8 years. ESSDAl at baseline was $6.62 \pm 6.37$ and currently is $4.21 \pm 5.16$ $(p<0.05)$. The mean of the SSDDI was $2.16 \pm 1.60$ and ESSPRI 8.38 \pm 6.88 . SGB was positive in $81.5 \%$ and focus score mean was 1.58 \pm 1.30 . Schirmer test I was positive in $72.6 \%$, van Bijsterveld in $70.9 \%$ and UWSF in $84.8 \%$. About classification criteria, 91.9\% fulfilled AECG 2002 and 94\% ACR/EULAR 2016. Anti-Ro and anti-La were positive in $70.8 \%$ and $35.5 \%$, respectively. Forty three percent had positive RF, $88.5 \%$ ANA, $6.9 \%$ low C3, $10.2 \%$ low C4, 34.9\% high lgG. Nineteen percent were using prednisone, $40.4 \%$ immunosuppressant, $53.6 \%$ antimalarial and $17.6 \%$ biological therapy. Prevalence of cardiovascular event was $7.5 \%$, hypertension $43.3 \%$, diabetes $13.3 \%$, dyslipidemia $31.5 \%$, smoking $5.8 \%$ and cancer $7.5 \%$.

Conclusion: ESSDAl has decreased over time and more than half of patients are on hydroxychloroquine, immunosuppressant or biological therapy. Further analysis is needed to understand whether the reduction in ESSDAI reflects the natural course of the disease or greater access to 